\title{
Requerimientos decalidad en el ejercicio de la mediación familiar licitada en Chile. Reflexiones para su conceptualización
}

\author{
Caterine Valdebenito Larenas \\ Universidad Central de Chile, Santiago, Chile. \\ Email: cvaldevenitol@ucentral.cl
}

\begin{abstract}
Resumen: Desde 2006 se instala la calidad en el ejercicio de la mediación familiar administrada por el Estado en Chile, siendo objeto de un conjunto de reglamentaciones orientadas a procesos de monitoreo y control ya la valoración del desempeño profesional de los/as mediadores/as en la sala de atención conforme a ciertos criterios conocidos. Este artículo revisa las teorías vigentes y su aplicación en el sistema de mediación familiar chileno tendiente a la construcción de lineamientos propios que aborden la calidad de las prestaciones ofrecidas. Desde una aproximación cualitativa / descriptiva y en base a la revisión de autores e investigaciones recientes, que permitan la construcción y el análisis de variables que den cuenta de la complejidad del proceso evaluativo. Entre los resultados se presenta una propuesta de modelo conceptual de la calidad de la mediación familiar, donde un tercer observador mediador problematiza la atención entregada y asimismo contribuye a la complejidad reflexiva.
\end{abstract} luación

Palabras claves:Mediación familiar, calidad de servicio, supervisión, eva-

\section{Quality requirements in the exercise of family mediation tendered in Chile. Reflections for its conceptualization}

\begin{abstract}
Quality has been set up since 2006 in the exercise of family mediation manage by the State of Chile, being the object of a set of regulations aimed at monitoring and control processes and the assessment of the professional performance of the mediators in the courtroom according to certain known criteria. This article reviews the current theories and their application in the Chilean family mediation system tending towards the construction of its own guidelines that address the quality of the services offered. From a qualitative / descriptive approach and based on the review of authors and recent research, which allow the construction and analysis of variables that account for the complexity of the evaluation process. Among the results, a conceptual model of the quality of family mediation is proposed, where a third mediating observer problematizes the attention given and also contributes to the reflexive complexity.
\end{abstract}

Keywords: Familiar mediation, quality of service, supervision, evaluation 


\section{Requisitos de qualidade no exercício da mediação familiar licitada no Chile. Reflexões para sua conceituação}

Resumo: Desde 2006, se encontra instalada a qualidade no exercício da mediação familiar administrada pelo Estado no Chile, sendo objeto de um conjunto de regulamentos destinados a processos de monitorização e controle e à avaliação do desempenho profissional dos/das mediadores/as na sala de cuidados sob certos critérios conhecidos. Este artigo analisa as teorias atuais e sua aplicação no sistema de mediação familiar chileno que possibilitem construir diretrizes próprias que abordam a qualidade dos serviços prestados. A partir de uma abordagem qualitativ / descritiva e com base na revisão de autores e pesquisas recentes, que permitam a construção e análise de variáveis que expliquem a complexidade do processo de avaliação. Entre os resultados se apresenta uma proposta de um modelo conceitual para a qualidade da mediação familiar, onde um terceiro observador mediador problematiza os serviços prestados e também contribui para a complexidade reflexiva. avaliação

Palavras-chave: mediação familiar, qualidade de serviço, monitoramento,

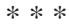

\section{Antecedentes Generales}

Desde el año 2005, la Mediación en temas de familia ha sido parte sustantiva de la política de justicia en Chile, con enfoque de equidad, la colocó dentro de las tareas que deben ser cubiertas por el Estado, de modo de asegurar la cobertura del servicio a nivel nacional.

Lo que se ha logrado bajo la política pública de contratación vía licitaciones públicas de servicios de mediación familiar, habiéndose desarrollado a la fecha, cuatro de estos concursos públicos.Tarea que, debe ser cumplida, conforme los estándares de prestación de servicios, regulados por la Unidad de Mediación del Ministerio de Justicia (en adelante UMED), que tiene la labor de la administración del servicio de mediación familiar.

Para ello, y conforme a la normativa vigente y el estado de instalación del nuevo programa, en los primeros años se trabajó en el monitoreo y control de los centros de mediación contratados ${ }^{1}$, de modo de unificar las condiciones de prestación de los servicios a nivel país. Donde, se comenzó paulatinamente a colocar el foco del quehacer de control en la manera de desarrollar la labor de mediación en la sala de atención. Ya en el año 2008 en adelante, fue posible abordar a desarrollar una política de corte más cualitativa, que permitió instalar la reflexión acerca de las características que debe disponer un proceso de mediación familiar, conforme la realidad chilena. Lo que se inició con un trabajo en torno a casos clínicos, siendo considerado un aporte por los operadores del sistema. 
Desde el año 2011, con seis años de funcionamiento el sistema de mediación familiar licitada (en adelante SNM), desde el ente regulador se trabajó en la línea de la confección de un sistema de evaluación de la calidad del servicio de la mediación familiar, considerando específicamente el trabajo realizado por el mediador con las partes en conflicto, en la sala de mediación. Lo que se concretó vía contratación de servicios de universidades.

\section{Metodología de supervisión ECAME: Evaluación de la calidad de la mediación}

El instrumento cuantitativo de Evaluación de la Calidad de la Mediación familiar, conocido como pauta ECAME, construido en el año 2011 por el equipo metodológico de CEAL - PUCV con fondos del Ministerio de Justicia, en un enfoque eminentemente práctico, construyó una definición de corte operativa que declaró:

Un proceso de resolución de conflictos regulado por principios dogmáticos y legales (componente legitimidad) en el cual, un mediador imparcial, genera comunicación efectiva(componente comunicación) y constructiva entre las partes y promueve la participación igualitaria (componente participación), autónoma y responsable en la búsqueda de soluciones a conflictos (componente resolutivo) familiares, facilitando la generación de acuerdos construidos desde las partes interesadas con recíproca satisfacción subjetiva(CEAL-PUCV, 2011, p. 6).

La definición operativa presentada, contiene las cuatro variables medidas cuantitativamente por el instrumento que fue aplicado a nivel nacional entre los años 2011 a 2014, en dos oportunidades, las que son: a) Legitimidad, definido como las prácticas asociadas a la legitimidad de los procesos de mediación familiar de acuerdo a principios legales y dogmáticos; b) Proceso Comunicacional, definido como las prácticas que permiten el entendimiento y la interacción positiva entre las partes en un proceso de mediación familiar; c) Proceso Participativo, definido como las prácticas que permiten la participación activa e igualitaria de las partes en el proceso de mediación familiar y d) Proceso Resolutivo, definido como las prácticas que permiten un procedimiento colaborativo de resolución de conflictos familiares.

El cuadro siguiente muestra las variables y sus respectivas dimensiones de análisis según el informe de la CEAL - PUCV: 


\section{Tabla 1}

Resumen operacional del ECAME

\begin{tabular}{|ll|}
\hline VARIABLES & DIMENSIONES \\
\hline Legitimidad & Transparencia. \\
& Igualdad (equilibrio de poder) \\
& Voluntariedad. \\
& Confidencialidad. \\
& Imparcialidad/Neutralidad. \\
& Interés superior del niño. \\
& Legalidad. \\
\hline Proceso & Comunicación efectiva. \\
Comunicacional & Comunicación constructiva. \\
\hline Proceso Participativo & Igualdad en la participación \\
& Protagonismo/Responsabilización. \\
\hline Proceso Resolutivo & Conducción del procedimiento. \\
& Colaboración en la Resolución. \\
\hline
\end{tabular}

Fuente: CEAL- PUCV. Auditoría Externa para la Evaluación de la calidad de los Procesos de Mediación Familiar. Informe Final definitivo. Septiembre 2011.

Dichas variables y sus dimensiones, recogidas en la pauta de evaluación validada estadísticamente permitieron dar cuenta de los ámbitos evaluados, según el grado de cumplimiento alcanzado por los profesionales que fueron evaluados en dichas oportunidades. Con resultados adecuados $^{2}$ a nivel país. Sin embargo, no se encontró la definición teóricoconceptual que debíasustentar este instrumento, y que es necesaria para la identificación rigurosa de los ejes temáticos explicitados, y por ello es que se pretende abordar reflexivamente en este documento.

\section{Revisión del concepto Calidad a la luz de los modelos clásicos}

Para la identificación de los elementos de la calidad de un proceso de atención en mediación familiar en el sistema de justicia chileno, fue necesario considerar cuales son los objetivos que se buscaron con la instalación de esta metodología de evaluación de la gestión de conflictos, los que de acuerdo a la UMED están en la necesidad de acercar la justicia a la ciudadanía, mediante un sistema previo al tribunal, que permita a las personas poder avanzar en la búsqueda de soluciones adecuadas a la realidad personal y familiar en que se encuentran, acorde al marco normativo y legal vigente desde el enfoque de mejoramiento continuo. 
La Ley Nº 19.968 en el Título V, artículo 103, definió lo que entiende como mediación familiar: aquel sistema de resolución de conflictos en el que un tercero imparcial, sin poder decisorio, llamado mediador, ayuda a las partes a buscar por sí mismas una solución al conflicto y sus efectos, mediante acuerdos.

Dicha definición, contiene dos propósitos de la política pública declarados en el texto del Mensaje Presidencial del Proyecto de Ley para la Creación de los tribunales de Familia ${ }^{3}$ como son en primer lugar, el conflicto familiar es un conflicto que reclama soluciones cooperativas, es decir, soluciones que acrecienten el bienestar de todas las partes del conflicto y con dicho fin, el proyecto instituye la mediación. La mediación, al aumentar los niveles de información disponibles y los canales de comunicación entre las partes, fomenta las soluciones auto compositivas.

Y en un segundo lugar, existe evidencia empírica de que este tipo de soluciones disminuye las ineficiencias, permitiendo así, en el largo plazo, un ahorro de recursos. La solución adversarial -al concebir el conflicto como una relación no interactiva tiende a asignar bienestar a una de las partes, negándoselo a la otra. Ello suele ser motivo de un nuevo conflicto que, muy prontamente, comparece de nuevo ante el sistema reclamando solución.

Por lo que colocó el énfasis en crear la institucionalidad básica que proporcionaría a las partes, instancias adecuadas para llegar a soluciones cooperativas, apuntando de esta manera a soluciones pacificadoras que favorecerían la armonía del grupo familiar y la consecución de resultados más favorables y adecuados para la resolución de los conflictos legales de la familia, que atañen a los niños, niñas y adolescentes en especial.

Lo anterior, asimismo consideró la necesaria eficacia y eficiencia que debe presentar toda política pública, destinada a optimizar los recursos al momento de acrecentar el acceso a la justicia de sectores tradicionalmente excluidos. Esto hace que el concepto de resultados en mediación familiar, entendido como los acuerdos alcanzados por las personas en el tema que dio origen a la mediación, tenga relevancia en la mirada de evaluación del uso de los recursos del Estado, tal como se desprendió de la última frase de la definición legal.

Ahora bien, dentro de la enunciación que colocó la ley N⒚968, se inició con la tesis de un sistema colaborativo de resolución de conflictos. Mirando los componentes de este sistema proceso de mediación, fue posible distinguir actores o personas participantes y que se encontrarían identificadas en la ley chilena: el tercero imparcial llamado mediador y las personas que viven la situación de conflicto que va ser atendida, lo que también se encuentra presente en los distintos modelos de la mediación.

Las personas, al ser quienes afrontarían el problema en crisis, traen consigo la historia de la vivencia del conflicto, sus manifestaciones, pérdi- 
das, ganancias y proyecciones de solución, conforme a sus propios intereses y necesidades y el entorno que los rodea. Cada historia es única y específica, y se denomina la narrativa del conflicto ${ }^{4}$ y es contada dentro del proceso de mediación con el uso de las técnicas y herramientas propias, permitiendo la construcción de un escenario distinto al inicial que trae cada persona y por ello, factible de ser intervenido en vistas a la búsqueda de nuevas soluciones que pueden ser visibilizadas por los propios sujetos afectados, en el propio marco social y cultural.

Clave en ello, van a ser los recursos personales, educacionales y de proyectos de vida futura que dispondrían las personas en conflicto, para la búsqueda de posibilidades de soluciones viables, distintas y novedosas a las ya intentadas. La disponibilidad de redes de apoyo también juega un papel relevante para la viabilidad de las soluciones en el tiempo.

El mediador es un profesional ajeno y externo a la realidad en conflicto, y cuya función primordial es, ayudar a las personas a buscar sus propias posibles soluciones, a lo que debemos agregar, en coherencia con la mirada sistémica de la mediación, la consideración de la realidad personal, familiar y social en que se encuentran.

La condición de "sin poder decisorio del mediador o mediadora”, está planteada como consecuencia desde el mensaje presidencial, que expuso: las partes involucradas pueda llegar por si mismas a acuerdos satisfactorios para ambas...comprometer personal y espontáneamente a las partes tenderán a sentar las bases de una relación pacifica en el futuro (Debate Parlamentario de la Ley 19.968 que Crea los Tribunales de Familia: s.f).Así recogió la importancia que colocó el legislador en el uso de herramientas que tienden a la autocomposición de los afectados y afectadas. Y ubicó expresamente la imposibilidad del profesional en tomar decisiones, ofrecer consejo profesional u otra conducta de corte asistencial que normalmente desarrollan las profesiones de ayuda a personas.

De modo de lograr las condiciones necesarias para el desarrollo del proceso de mediación, donde los actores internos pudiesen desenvolverse para el logro del objetivo de alcanzar acuerdos, la ley, recogiendo la experiencia comparada, enmarcó el ejercicio de la mediación en torno a principios de corte dogmático y normativa legal ${ }^{5}$ que deberían estar presentes en todo el quehacer mediador y que correspondieron a igualdad, voluntad de participación de las partes, confidencialidad e imparcialidad, los que se están presentes en los distintos enfoques teóricos de los sistemas de gestión colaborativa de conflictos (Suares: 2012; Bush y Folger: 2006, Folger: s.f, Parkinson: 2005; Ury: 2012). 
Tabla 2

Principios según los modelos de mediación familiar

\begin{tabular}{|llll|}
\multicolumn{1}{c}{$\begin{array}{c}\text { Modelo } \\
\text { de Harvard }\end{array}$} & \multicolumn{1}{c|}{$\begin{array}{c}\text { Modelo Circular } \\
\text { Narrativo }\end{array}$} & \multicolumn{1}{c|}{$\begin{array}{c}\text { Modelo } \\
\text { Transformativo }\end{array}$} & \multicolumn{1}{c|}{$\begin{array}{c}\text { Modelo } \\
\text { Ecosistémico }\end{array}$} \\
\hline - Imparcialidad & -Confidencialidad & -Confidencialidad & -Confidencialidad \\
-Confidencialidad & -Equilibrio de & -Equilibrio de & -Equilibrio de \\
-Equilibrio de & poderes & poderes & poderes \\
poderes & -Imparcialidad & -Voluntad de las & -Voluntad de las \\
-Autocomposición & -Neutralidad & partes & partes. \\
-Mirada de futuro & -Ideología de & & -Interés superior \\
& futuro & & de los NNA \\
& & & -Co-parentalidad \\
& & & -Género \\
& & & -Mirada de futuro. \\
\hline
\end{tabular}

Fuente: elaboración propia

Y agregó de manera explícita dos ejes rectores como son el interés superior del niño y las opiniones de terceros (Córdova, González y Valdebenito: 2012, p. 162), que dan cuenta del especial interés por relevar la figura de los niños, niñas y adolescentes como sujetos de derecho, que deben ser considerados en todo momento del proceso de la mediación. Los que también se encuentran explicitados en el modelo Ecosistémico según se puede observar en la tabla 2.

Los principios descritos, se entienden presentes y desplegados de forma permanente en la conducta y actuación profesional del mediador, independientemente del momento de trabajo del proceso de mediación, acorde a la temática especifica que se encuentre en atención y el marco legal vigente, asegurando con ello el desarrollo de prestaciones en un marco de ética profesional acorde a la idea de calidad de servicio.

\section{Calidad en las etapas de atención de la mediación familiar}

De modo de avanzar en el conocimiento y aplicación de los principios y funcionamiento de la mediación familiar en Chile, la UMED, acorde los resultados propuestos por el plan piloto de los centros de mediación anexos a tribunales, estableció la necesidad de ordenar los contenidos mínimos que deberían de estar presentes en el desempeño del mediador en un proceso de mediación en temas de familia; labor que ha ido desarrollando y complementando de manera progresiva tal como ya se indicó.

La fase inicial de la mediación, favorecería la aplicación del instrumento llamado discurso inicial ordenándolo en torno un documento llamado Consentimiento Informado, el que detalló la información sobre mediación familiar necesaria de entregar a las personas que asisten a mediación. 
La pauta ECAME del año 2011, colocó en el punto de Legitimidad, la apreciación de este aspecto, estableciendo un sistema de mediación mediante la evaluación de la explicación que realizaría el mediador o mediadora de cada uno de los principios básicos y legales que ordenan el sistema de mediación licitada, los que se evalúan en condiciones de si son señalados o no.

Parece necesario mirar la complejidad de la aplicación de los principios rectores, además de la consideración de incorporación de la intersectorialidad de derechos que deben guiar los procesos de trabajo en mediación, donde el foco se encontró en la relación que establece el mediador o mediadora con los y las sujetos atendidos tendiente a apoyar el mejoramiento de los canales de comunicación existentes, de modo de facilitar la búsqueda de posibles soluciones acordadas entre éstos.

Es adecuado poder ampliar el sentido de la evaluación de la aplicación de los principios dogmáticos y legales, no solo a la sesión inicial -que ya posee un sistema de control en el consentimiento informado- sino a la aplicación por parte del mediador en todo momento del proceso de la mediación, mediante la observación de conductas y verbalizaciones que realice el profesional cuando sea procedente en la atención que ejecuta independiente del enfoque teórico, con un enfoque de evaluación del proceso de trabajo que realizó, en sintonía con el enfoque sistémico que impregna la definición legal de mediación familiar.

Otro aspecto que contempló la definición de la normativa chilena sobre mediación, se vinculó con la aseveración que el mediador ayuda a las partes a buscar por sí mismas una solución al conflicto y sus efectos. Donde aparece necesario acordar el alcance de la noción de ayuda para la búsqueda de soluciones, en un marco limitado de accionar profesional.

Avanzando en la teoría sobre mediación, desde la mirada del modelo transformativo (Bush y Folger, 2006, p.130), no se definió la noción de ayuda del mediador. Sino por el contrario, declaró que el aporte de esta mirada teórica está en ayudar a las personas a transformar sus dimensiones morales.

Aportó en indicar que los resultados de las técnicas de revalorización de sí mismo y el reconocimiento del otro, solo serán posibles desde el trabajo de fortalecimiento de la conciencia del yo en el ámbito de las sesiones de mediación, pudiendo ser observados en la actitud de cada persona respecto de sí mismo y respecto del otro, más allá de haber o no alcanzado acuerdos específicos en la mediación.

Para ello, colocó el foco en la observación y análisis de las pautas comunicacionales y actitudinales existente entre las personas en conflicto, en las que interviene desde un micro-enfoque, centrado en la transformación de las pautas interpersonales entre los sujetos, con la convicción que el mediador con sus intervenciones de corte comunicacional y actitudinal, 
aportaría en apoyar la exposición de la vivencia del conflicto y la comprensión de las perspectivas de cada uno de los y las involucrados.

El modelo circular narrativo (Sluzki, 2011,p. 6), coloca como función de ayuda del mediador la modalidad de trabajo de de-construcción del conflicto traído a mediación, para la construcción de una nueva narrativa, utilizando para ello, la metodología de involucración y neutralidad (Suares, 2005. P. 278-280) del profesional.

Este forma de abordaje profesional, colocó la mirada en las narrativas de las partes del conflicto, donde una de ellas habría colonizado el relato de conflicto, por lo que el mediador abordaría prestando atención a los sub-relatos de cada una de las historias, para la deconstrucción del conflicto y la búsqueda de historias alternativas que permitiesen en forma compartida, alcanzar una forma ampliada de entender la problemática y movilizar nuevas herramientas en los sujetos, de modo de avanzar en la búsqueda de soluciones a la vivencia conflictiva que comparten. Y la noción de ayuda del mediador, pasó a tener la perspectiva de una intervención profesional desde las teorías comunicativas y narrativas, colocando el énfasis en las herramientas pragmáticas y reflexivas de la comunicación humana.

El modelo Ecosistémico (Parkinson, 2005, p. 80), recogió la noción de la mediación familiar, como un proceso de intervención, destinado a apoyar a los sujetos en crisis a manejar los cambios y la comunicación entre ellos para la toma de decisiones hacia el futuro, en este tiempo de transición y reajustes por la crisis vivida.

Por ello, postuló como ejes de intervención profesional por parte del mediador, el conocimiento del conflicto y sus repercusiones en el sistema familia y el entorno inmediato, buscando el fortalecimiento de los y las sujetos directamente afectados para la toma de decisiones en los ámbitos de disputa.

Lo que permitiría que el mediador despliegue la metodología de acompañamiento flexible a las partes en conflicto, que permitirían el uso de herramientas de comunicación destinadas a compartir, traducir e interpretar los relatos, de modo de apoyar la cooperación entre los integrantes de la familia, en un espacio de acogida y de contención a la crisis que enfrentan. Las habilidades y competencias de tipos comunicativas y sociales, pasan a ser centrales para que el mediador desarrolle el proceso de mediación familiar.

El modelo de Harvard (Fisher y Ury, 2012, p. 10), entendió la noción de ayuda del profesional, en la línea funcionalista de avanzar desde las posiciones a los intereses y necesidades subyacentes de las personas en conflicto respecto del tema en disputa, mediante la utilización de la metodología de la negociación asistida, con una figura de mediador como un actor que concentraría atención y que apuntaría a problematizar a las partes para la búsqueda de soluciones. 
Desde esta perspectiva, el mediador tiene a su disposición la batería de metodologías de la negociación colaborativa, aportando desde lo lingüístico y analógico a la resolución de la disputa en particular.

\section{Tabla 3}

\section{Enfoque e intervenciones según los modelos de mediación familiar}

\begin{tabular}{|c|c|c|c|}
\hline Modelo de Harvard & $\begin{array}{l}\text { Modelo Circular } \\
\text { Narrativo }\end{array}$ & $\begin{array}{c}\text { Modelo } \\
\text { Transformativo }\end{array}$ & Modelo Ecosistémico \\
\hline $\begin{array}{l}\text { Línea funcionalista de } \\
\text { avanzar desde las } \\
\text { posiciones a los } \\
\text { intereses y } \\
\text { necesidades } \\
\text { subyacentes de las } \\
\text { personas en conflicto } \\
\text { respecto del tema en } \\
\text { disputa, mediante la } \\
\text { utilización de la } \\
\text { metodología d la } \\
\text { negociación asistida }\end{array}$ & $\begin{array}{l}\text { Trabajo de } \\
\text { deconstrucción del } \\
\text { conflicto traído a } \\
\text { mediación, para la } \\
\text { construcción de una } \\
\text { nueva narrativa, } \\
\text { utilizando para ello, la } \\
\text { metodología de } \\
\text { involucracióny } \\
\text { neutralidad }\end{array}$ & $\begin{array}{l}\text { Foco en la } \\
\text { observación y análisis } \\
\text { de las pautas } \\
\text { comunicacionales y } \\
\text { actitudinales existente } \\
\text { entre las personas en } \\
\text { conflicto, en las que } \\
\text { intervendrá desde un } \\
\text { micro-enfoque, } \\
\text { centrado en la } \\
\text { transformación de las } \\
\text { pautas interpersonales } \\
\text { entre los sujetos, }\end{array}$ & $\begin{array}{l}\text { Proceso de } \\
\text { intervención, } \\
\text { destinado a apoyar a } \\
\text { los sujetos en crisis a } \\
\text { manejar los cambios y } \\
\text { la comunicación entre } \\
\text { ellos para la toma de } \\
\text { decisiones hacia el } \\
\text { futuro, en este tiempo } \\
\text { de transición y } \\
\text { reajustes por la crisis } \\
\text { vivida. }\end{array}$ \\
\hline
\end{tabular}

Fuente: elaboración propia

La tabla 3 permitió observar de forma comparada las diferencias y semejanzas existentes en la forma de abordaje del conflicto entre los modelos descritos, aportando en la selección de los hitos de calidad interna de cada uno de ellos. El foco de trabajo entonces, se encontró en avanzar en la identificación de las áreas en disputa, la comprensión de los significados que cada parte le otorgaría, para así apoyar en colocar en común los puntos de conflictos y sus implicancias para la búsqueda de soluciones.

De acuerdo las teorías y modelos descritos, es posible concluir que la idea de ayuda/ facilitación del mediador que estaría dentro de la definición legal de la mediación familiar, se refiere al rol del desempeño profesional que puede tomar un profesional mediante el uso de herramientas y destrezas comunicativas, de conocimiento y actitudinales que favorezcan la aplicación de los principios rectores de la mediación, de modo de colaborar en el ejercicio de las personas en conflicto en la búsqueda de posibles soluciones que les permitan alcanzar eventualmente acuerdos.

Las competencias de conocimiento, comunicativas y actitudinales que posean los mediadores, constituyen elementos visibles dentro del proceso de trabajo de cada mediación familiar, y que son desplegados por el profesional, en consideración de las características de cada causa en atención: la problemática en crisis, la historia del conflicto, los actores involucrados, los recursos internos y externos disponibles, las condicio- 
nes del entorno, como parte de los elementos necesarios de considerar dentro de la intervención profesional a desarrollar.

Ello implicaríala necesidad de identificar las herramientas comunicativas de acogida, de contención emocional, de escucha activa y técnicas reflexivas que aportan al trabajo de la mediación en temas de familia.

Asimismo, requiere de la caracterización de la batería de herramientas destinadas a favorecer la participación voluntaria y activa de las personas en el proceso de gestión y resolución del problema en cuestión, mediando el aprendizaje de esta metodología en las personas, como elemento que en un futuro, les puedan apoyar en la búsqueda de soluciones a sus dificultades.

Este ideario, se recogió en el instrumento ECAME, bajo la forma de dos componentes, como son el componente comunicacional y el componente participativo, los que abordaron la identificación de un conjunto de conductas deseables que sean desarrolladas por el mediador familiar. De esta forma, y bajo el prisma de la observación participante, se privilegió la aplicación de habilidades y técnicas que posibiliten la presencia activa de las personas en la mediación, en un escenario de acogida, contención y progresivo empoderamiento construido en la relación profesional-personas en conflicto, como parte del proceso de atención afrontado.

\section{Figura 1 \\ Criterios de intervención de calidad según los modelos de mediación familiar}

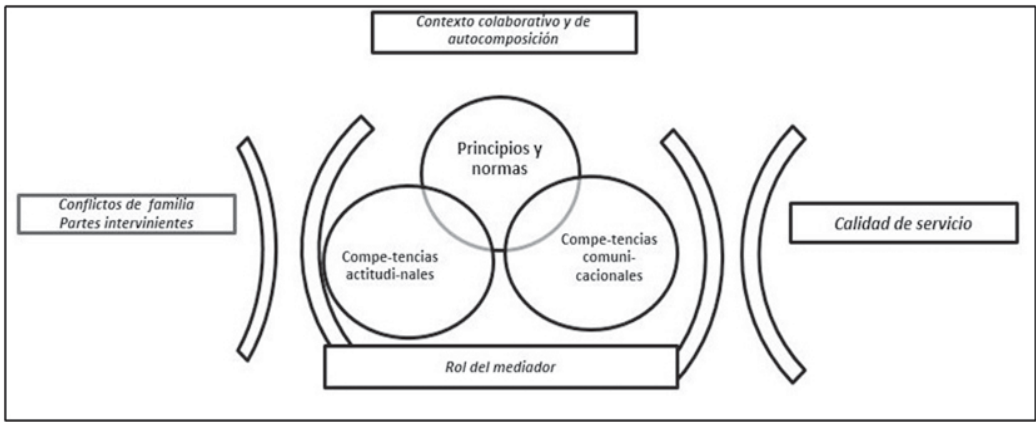

Fuente: Elaboración propia

La figura 1 dio cuenta de la mirada compleja que significa la incorporación de la noción de calidad al proceso de atención en mediación, que entremezcla aspectos del desempeño profesional en la gestión de los con- 
flictos familiares, en un escenario más amplio que incluye el contexto legal y normativo de colaboración y autocomposición.

La complejidad de la atención de un proceso de mediación familiar tiene como telón de fondo, la construcción de un trabajo profesional de tipo colaborativo cuyos resultados finales dependen de la voluntad y capacidades de los actores principales del conflicto; por lo que la concepción de la mediación como un proceso de trabajo, debe estar presente en una mirada de evaluación de la calidad de servicio, privilegiando un adecuado desempeño que apoye la búsqueda de posibles soluciones a los conflictos afrontados.

El gobierno y resolución del conflicto, mediante el uso de un conjunto de herramientas de tipo analítico y reflexivo, acorde a los recursos y perfiles de las personas en atención, debe constituir el foco de cierre de un proceso de mediación. De modo que sean los propios sujetos, los que en consecuencia del proceso comunicativo y de fomento de la participación colaborativa vivido dentro de la sala de mediación, avancen en la gestión y reordenamiento de las vivencias de crisis en tratamiento, con un enfoque de mejoramiento de la situación, que les permita avanzar en la valoración de sí mismos como personas y el reconocimiento de la contraparte como parte de la solución del problema.

Habiendo llevado de forma plena y responsable la conducción de un proceso de mediación, el mediador responsable, podrá evaluar con éxito el término de la misma, pudiendo valorar no solo la existencia de un acuerdo alcanzado en la temática en conflicto, sino también la existencia de logros intermedios cuando estos existan.

\section{Mapa conceptual de la definición de la calidad de la mediación familiar}

A continuación, y de manera gráfica, se expone la relación de los conceptos claves en la definición conceptual de la calidad de la mediación en temas de familia presentada en los párrafos anteriores, considerando las bases planteadas desde el instrumento inicial, y los avances alcanzados en la actualización del mismo con la revisión de los modelos teóricos más relevantes. 


\section{Figura 2}

Mapa conceptual de la calidad de mediación familiar

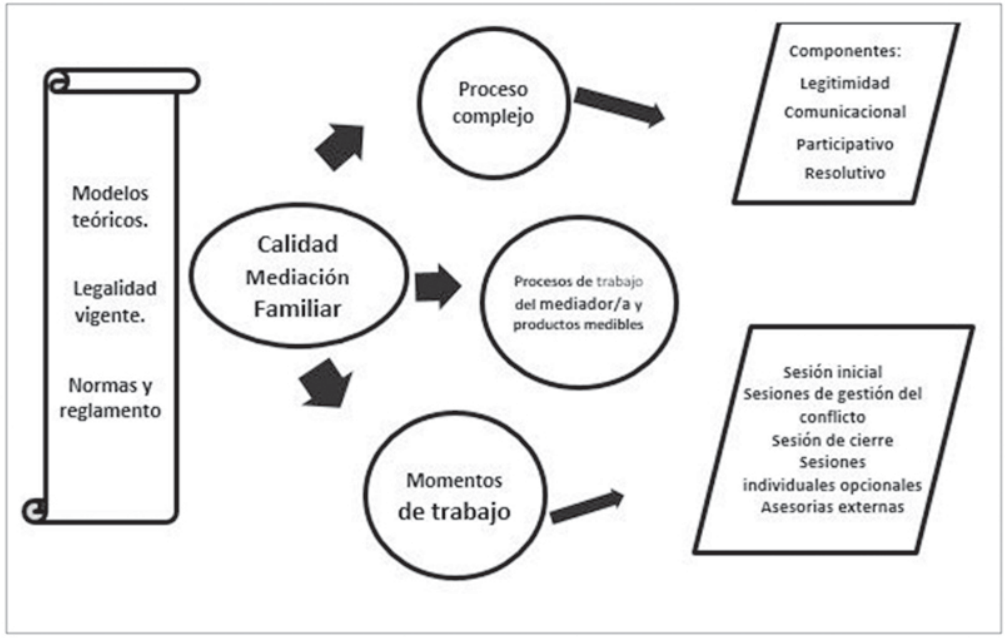

Fuente: Elaboración propia.

Con la base conceptual articulada y enriquecida, para la autora es posible ahora, proponer una definición operativa y actualizada de Calidad de la mediación familiar, posible de ser medida de forma cuantitativa y cualitativamente:

"Un proceso de gestión de conflictos regulado en todo su desarrollo, por un conjunto de principios y normativasdonde un profesional mediador imparcial, modela y promueve conductas de comunicación efectiva y constructiva entre las personas y fomenta la participación activa, autónoma y responsable en la búsqueda de posibles alternativas de soluciones a las manifestaciones de las crisis familiares, facilitando la edificación de posibles soluciones que constituyan acuerdos construidos desde las partes interesadas con recíproca satisfacción”

Lo anterior,actualizó en tres aspectos claves la definición base del ECAME propuesto por la CEAL - PUCV en el año 2011 y que son: a) La modernización del componente legitimidad, considerando su presencia, en todo momento del proceso de mediación, b) La modernización del componente resolutivo, considerando que la gestión del conflicto, debe entenderse como un componente de trabajo de mayor duración y complejidad, pudiendo mostrarse en diferentes momentos de la mediación y no solo al cerrar el proceso y c) La evaluación centrada en conductas y actitudes del profesional mediador que posibiliten y/o potencien los espacios y escena- 
rios de comunicación efectiva y de participación activa de las personas que enfrentan la situación de conflicto en tratamiento en la mediación, por sobre la respuesta de un acuerdo de los sujetos en particular.

Con ello se buscóla consideración global de la situación, los actores y tipología del conflicto existente, por sobre la aplicación y utilización de determinadas herramientas por parte del mediador, en el marco mayor del respeto a los derechos humanos y garantías procedimentales que los resguarden.

Estos desempeños profesionales, deben ser revisados a la luz de los límites de la práctica profesional (Cruz, 2008), de modo de salvaguardar la individualidad del sujeto mediador y la individualidad de las partes que asisten a mediación, de modo de prevenir situaciones de desgaste emocional innecesario entre los intervinientes como su propio desgaste profesional.

Todo ello, bajo la garantía de respeto a la diversidad en el ejercicio profesional, considerando, no solo el cumplimiento de normas vigentes, sino por sobre todo, la evaluación integral del proceso de trabajo desarrollado, conforme a la temática abordada y las particularidades de cada grupo participante.

\section{Tabla 4}

\section{Ejes de la calidad según los modelos de mediación familiar y el ECAME}

\begin{tabular}{|c|c|c|c|c|}
\hline Modelo de Harvard & $\begin{array}{c}\text { Modelo Circular } \\
\text { Narrativo }\end{array}$ & $\begin{array}{c}\text { Modelo } \\
\text { Transformativo }\end{array}$ & $\begin{array}{c}\text { Modelo } \\
\text { Ecosistémico }\end{array}$ & Pauta ECAME \\
\hline $\begin{array}{l}\text {-Con base en } \\
\text { negociación } \\
\text { colaborativa. } \\
\text {-Énfasis en contenido } \\
\text { del problema } \\
\text {-Principios de } \\
\text { imparcialidad, } \\
\text { confidencialidad, } \\
\text { equilibrio de poderes, } \\
\text { autocomposición y } \\
\text { mirada de futuro } \\
\text {-4 etapas sucesivas y } \\
\text { complementarias de } \\
\text { trabajo centradas en el } \\
\text { contenido del problema. } \\
\text {-Producto esperado es la } \\
\text { solución acordada y } \\
\text { escriturada }\end{array}$ & $\begin{array}{l}\text {-Narrativa de las } \\
\text { personas. Enfasis } \\
\text { en las pautas } \\
\text { comunicacionales } \\
\text { existentes. } \\
\text {-Principios de } \\
\text { confidencialidad, } \\
\text { equilibrio de } \\
\text { poderes, } \\
\text { imparcialidad, } \\
\text { neutralidad e } \\
\text { ideologia de } \\
\text { futuro } \\
\text {-5 etapas } \\
\text { complementarias } \\
\text { de trabajo } \\
\text { centradas en la } \\
\text { vivencia del } \\
\text { conflicto. } \\
\text {-Producto } \\
\text { esperado es una } \\
\text { nueva narrativa } \\
\text { co-construida }\end{array}$ & $\begin{array}{l}\text {-Posibilidad de } \\
\text { cambio de las } \\
\text { personas y la } \\
\text { construcción de } \\
\text { una sociedad más } \\
\text { justa } \\
\text {-Principios de } \\
\text { empoderamiento } \\
\text { del sujeto, } \\
\text { reconocimiento } \\
\text { del otro y la } \\
\text { concientización. } \\
\text {-Proceso } \\
\text { desarrollado en - } \\
\text { micro- } \\
\text { movimientos } \\
\text { centrados en la } \\
\text { construcción de } \\
\text { nuevas } \\
\text { percepciones y } \\
\text { cambios en la } \\
\text { relación de las } \\
\text { personas y con el } \\
\text { entorno } \\
\text { inmediato. } \\
\text {-Producto } \\
\text { esperado son las } \\
\text { relaciones } \\
\text { interpersonales } \\
\text { modificadas }\end{array}$ & $\begin{array}{l}\text {-Apoyo a los } \\
\text { miembros de la } \\
\text { familia a conocer, } \\
\text { manejar los cambios y } \\
\text { las pautas } \\
\text { comunicacionales } \\
\text { entre ellos. } \\
\text {-Principios de } \\
\text { confidencialidad, } \\
\text { equilibrio de poderes, } \\
\text { voluntad de las partes, } \\
\text { interés superior de los } \\
\text { NNA, coparentalidad, } \\
\text { genero y mirada de } \\
\text { futuro. } \\
\text {-3 etapas } \\
\text { complementarias de } \\
\text { trabajo centradas en la } \\
\text { estructuración del } \\
\text { nuevo grupo familiar. } \\
\text {-Producto esperado es } \\
\text { el reordenamiento de } \\
\text { las relaciones parento- } \\
\text { filiales en la nueva } \\
\text { estructura familiar }\end{array}$ & $\begin{array}{l}\text {-Proceso de gestión } \\
\text { de conflictos } \\
\text { regulado. } \\
\text {-Principios } \\
\text { dogmáticos de la } \\
\text { mediación y normas } \\
\text { legales el articulo } \\
107 . \\
\text {-Trabajo centrado } \\
\text { en promover: } \\
\text { +conductas de } \\
\text { comunicación } \\
\text { efectiva y } \\
\text { constructiva. } \\
\text { +la participación } \\
\text { activa, autónoma y } \\
\text { responsable de las } \\
\text { personas. } \\
\text { +facilitando } \\
\text { posibles alternativas } \\
\text { de soluciones que } \\
\text { constituyan } \\
\text { acuerdos } \\
\text { construidos desde } \\
\text { las partes. } \\
\text {-Producto esperado: } \\
\text { acta de mediación } \\
\text { escriturado }\end{array}$ \\
\hline
\end{tabular}

Fuente: elaboración propia 
La tabla 4, muestra los elementos propios de la calidad de los distintos modelos de mediación y los hitos que identificaría la pauta ECAME, que como se observa, contiene elementos de los distintos enfoques teóricos revisados, construyendo un esquema de evaluación de mínimos y máximos para la consideración de los resultados de la calidad del desempeño del mediador familiar, coherente con la declaración realizada por la UMED, de no adscribir a algún modelo de mediación en particular.

A modo de conclusión: el aporte de la supervisión con enfoque de pares para el mejoramiento de la calidad del servicio

La tarea de evaluar el desempeño de un mediador es un proceso complejo de realizar. Ya que se establece la tarea en un espacio de trabajo cerrado entre las personas que son atendidas y el profesional que las atiende, lo que es cautelado por el conjunto de principios que la regulan.

A ello debe sumársele, las reticencias que produce cualquier sistema de evaluación de desempeños en los trabajadores cada vez que son evaluados, lo que en el sistema público chileno se desarrolla anualmente. Modalidad que se quiere replicar en el SNM, con vistas al mejoramiento continuo de la calidad del servicio.

Sin embargo, en un estudio realizado a los y las mediadores familiares (Valdebenito, 2015, p. 155), se pudo conocer el interés que presentan de poder contar con un espacio reflexivo de supervisión, enfocándolo como parte de un dispositivo de capacitación práctica que apoye la reflexión que necesita cada caso atendido.

La importancia que le entregan los mediadores a esta herramienta, como un espacio de reflexión y de socialización de saberes en relación con las prácticas (Robles, 2010) y al mismo tiempo un intercambio entre pares, coordinado por un especialista y enriquecido con aportes bibliográficos para arribar a propuestas de acción, es lo que permite sea visualizada como un proceso de aprendizaje y no un sistema de control y evaluación.

Los criterios propios de la supervisión clínica son recogidos en este enfoque con vistas al mejoramiento de la calidad del servicio, destinados a observar y estudiar desde un enfoque global que permite tomar distancia del objeto-sujeto observado, de modo de potenciar a través de la orientación, educación y capacitación a los trabajadores directamente responsables de la ejecución de una actividad o proyecto específico. Y en el marco institucional provisto por el Estado, la colaboración de un experto designado para ese fin, de cara a lograr un mayor aprovechamiento de sus conocimientos y habilidades y perfeccionamiento de sus actitudes, posibilita el acceso a herramientas para ejecutar las tareas de una forma más eficiente y con una satisfacción cada vez mayor para ellos mismos y para la institución (Sallard, 2006, p. 48). 
La metodología ECAMEcon enfoque de supervisión entre pares aplicada en el estudio de 2013, sustenta parte de sus postulados en lo señalado por Sallard, que explicita que para que el proceso sea llevado en buena forma, es indispensable que los momentos que van a ser observados sean en acordados con los participantes, de modo de apoyar en la vivencia de exposición pública (Sallard, 2006, p. 62) que necesariamente acarrea este proceso.

Ello fue posible cuando se sumaron dos nuevos instrumentos de corte más cualitativo, como fueron la pauta de autoevaluación del mediador y la pauta de retroalimentación de responsabilidad del supervisor, con el objetivo de generar el espacio de conversación y reflexión de la práctica observada.

A nivel concreto, el supervisor comparte con el mediador el registro de sus impresiones técnicas acerca del trabajo observado en la sesión de mediación, cuidando la forma de su comunicación, privilegiando el componente pedagógico de esta devolución, con la finalidad que se estableciese un vínculo de enseñanza-aprendizaje entre ambos que favorezca la reflexión del mediadorsupervisado, en vistas al mejoramiento continuo de su desempeño profesional.

Es necesario señalar que en la aplicación de estos tres instrumentos, se acordó n el siguiente orden en su uso: primero la aplicación de la pauta ECAME, luego la completación de las dos pautas cualitativas de autoevaluación y de retroalimentación y finalmente la devolución de la pauta de retroalimentación del supervisor.

Finalmente, se hace necesario comentar que la devolución del proceso realizada por los mediadores familiares supervisados en ese período, reportó que el espacio de trabajo de devolución en sala por parte del supervisor respecto de la práctica mediadora observada, fue el elemento valioso dentro del trabajo realizado, ya que les permitió establecer un espacio introspectivo sobre su propio desempeño, iluminado por una mirada experta y reflexiva sobre la experiencia compartida, apoyando así la mirada de mejoramiento de la calidad como un proceso continuo y en desarrollo.

\section{Agradecimientos}

Artículo es parte sustantiva del marco referencial de la tesis para optar al grado de Master Executive Europeo y latinoamericano en Mediación y Negociación de APEP-IUKB en asociación con la Universidad de Ginebra, que fue aprobada en condición de Distinción Mayor, que corresponde a la más alta categoría en el $1^{\circ}$ semestre 2015. 


\section{Notas}

${ }^{1}$ La autora fue la responsable de la creación e implementación del área de Control e inspecciones de la Unidad de Mediación del Ministerio de Justicia, entre los años 2006 a 2008.

${ }^{2}$ Los estudios aplicados de la metodología ECAME están disponibles en la página del M. Justicia: www.mediacionchile.cl.

${ }^{3}$ Para profundizar este punto, se sugiere revisar el texto del Mensaje Presidencial en el documento Historia de la Ley $\mathrm{N}^{\circ} 19.968$, disponible en; www.leychile.cl/Navegar/scripts/ obtienearchivo.

${ }^{4}$ Este concepto, es propio del modelo circular narrativo de Cobb, de amplia difusión en Chile, y que ha sido aceptado y descrito por distintos autores, de modo de poder dar un espacio a reconocer y diagnosticar con las personas las manifestaciones del conflicto en el tiempo, las soluciones intentadas y los recursos que disponen para una solución.

${ }^{5}$ La Ley $\mathrm{N}^{\circ}$ 19.968, recoge en el artículo 107, los cuatro principios dogmáticos compartidos por distintos modelos de mediación: imparcialidad, confidencialidad, voluntad de participación de las partes y equilibrio de poderes entre las personas, a lo que agrega los dos preceptos legales de interés superior del niño/a y adolescente y la opinión de terceros, entendidos estos dos últimos como criterios relevantes en los temas de familia y que se describen en el texto. 


\section{Bibliografía}

Biblioteca del Congreso Nacional. (2015).Mensaje Presidencial, Documento Historia de la Ley $N^{\circ} 19.968$. Santiago, Chile. Recuperado de https:/ /goo.gl/95Hc4Q.

Bush, R. y Folger, J. (2006).La promesa de la mediación. Cómo afrontar el conflicto mediante la revalorización y reconocimiento. Buenos Aires, Argentina: Granica.

Córdova, J, González, I. y Valdebenito, C. (2012). Niños, niñas y adolescentes en la mediación. Bases de un modelo en construcción. En Memorias del VIII Foro Mundial de Mediación (I), (pp.159-165) Valencia, España.

Cruz. M. (2008).Crónica de la práctica de supervisión en Mediación Familiar,II Congreso de Mediación y Convivencia, Gandía- Valencia, España: Universitat Valencia.

Fisher R. Ury, W. y Patton, B. (2012).Si...de acuerdo. Buenos Aires, Argentina: Paidós.

Folger, J. (sin fecha). Mediación Transformativa: preservación del potencial propio de la mediación en escenarios de disputas. Recuperado de https://goo.gl/WaMM8T

Ley $\mathrm{N}^{\circ} 19968$ que crea los Tribunales de Familia, publicada el 30 de agosto de 2004. Recuperado de: http://www.leychile.cl.

Parkinson, L. (2005).Mediación Familiar. Teoría y práctica: principios y estrategias operativas, Buenos Aires, Argentina: Gedisa.

PUCV- CEAL (2011).Estudio sobre la calidad de la mediación familiar en Chile, Valparaíso. Recuperado de: www.mediacionchile.cl

Robles, C. (2010).Supervisar ¿Para qué? Lo oculto tras la resistencia. Buenos Aires, Argentina: Espacio.

Sallard. S. (2006).Mediación, supervisión y contención: Una visión tridimensional.Ciudad de México, México: Universidad de Sonora.

Sluzki, C. (2011). El camino desde el conflicto a la reconciliación. La coexistencia como proceso evolutivo.Buenos Aires, Argentina: Material para asistentes al Programa Internacional en Mediación Familiar

Suares, M. (2012).Mediación; conducción de disputas, comunicación y técnicas., Buenos Aires, Argentina: Paidós

Suares, M. (2005).Mediando en sistemas familiares. Buenos Aires. Argen- 
tina: Paidós.

Ury, W. (2012).Supere el no. Buenos Aires, Argentina: Editorial Norma.

Valdebenito, C. (2015).Mediación familiar en Chile. Un estudio sobre modelos y técnicas (Tesis de magíster no publicada),APEP-IUKB- en asociación con la Universidad de Ginebra. Buenos Aires, Argentina.

Recibido: 09.07.15

Aceptado: 27.06.17 